\title{
Exchange functional that tests the robustness of the plasmon description of the van der Waals density functional
}

\author{
Kristian Berland* and Per Hyldgaard ${ }^{\dagger}$ \\ Department of Microtechnology and Nanoscience, MC2, Chalmers University of Technology, SE-41296 Göteborg, Sweden
} (Received 9 September 2013; revised manuscript received 22 December 2013; published 13 January 2014)

\begin{abstract}
Is the plasmon description within the nonlocal correlation of the van der Waals density functional by Dion and coworkers (vdW-DF) robust enough to describe all exchange-correlation components? To address this question, we design an exchange functional based on this plasmon description as well as recent analysis on exchange in the large-s regime. In the regime with reduced gradients $s=|\nabla n| / 2 n k_{\mathrm{F}}(n)$ smaller than $\approx 2.5$, dominating the nonlocal correlation part of the binding energy, the enhancement factor $F_{x}(s)$ closely resembles the Langreth-Vosko screened exchange. In the $s$ regime beyond, dominated by exchange, $F_{x}(s)$ passes smoothly over to the revised Perdew-Wang-86 form. We term the specific exchange functional LV-PW86r, wheras the full van der Waals functional version emphasizing consistent handling of exchange is termed vdW-DF-cx. Our tests indicate that vdW-DF-cx produces accurate separations and binding energies of the S22 data set of molecular dimers as well as accurate lattice constants and bulk moduli of layered materials and tightly bound solids. These results suggest that the plasmon description within vdW-DF gives a good description of both exchange and correlation effects in the low-to-moderate $s$ regime.
\end{abstract}

DOI: 10.1103/PhysRevB.89.035412

PACS number(s): 31.15.E-, 31.15.A-, 61.50.Lt, 71.15.Mb

Van der Waals forces are essential for the properties of a wide range of materials and physical processes. Interesting examples go far beyond model cases such as noble gas dimers and include examples such as biomolecular matter, many transition-metal oxides, and chalcogenides. Even to fully describe many covalent effects, van der Waals forces are needed. For catalytic processes, it can be necessary to describe how molecules first adsorb on surfaces or within porous matter. In systems where covalent or electrostatic forces are the primary cause of binding, van der Waals forces sometimes tip the balance between competing configurations or even cause stronger chemical binding.

The lack of van der Waals forces in the extensively used generalized gradient approximations (GGAs) to density functional theory (DFT) have triggered many attempts to develop the theory beyond GGA in DFT, as well as attempts to extend related electronic-structure methods to include these forces. A conceptually simple and popular approach is to add pair potentials between the ionic centers of the atoms on top of GGA accounts [1]. These pair potentials may be empirical or semiempirical, that is, fitted once and for all on a given data set. Among the most well known formulations are those of Grimme and coworkers [2-4]. A more sophisticated method is to explicitly calculate, based on some scheme, the $C_{6}$ coefficients in a given system [5-8]. These kinds of methods remain in the atomic-pair potential paradigm, yet do contain some ability to adjust the dispersion account to the local environment, and they typically reduce the semiempiricism to a single parameter. The TS method by Tkatchenko and Scheffler is a prominent example [9].

The adiabatic connection formula (ACF) $[10,11]$ provides a formally exact determination of the interacting electron system. The ACF can be approximated within nonempirical

\footnotetext{
*berland@ chalmers.se

†hyldgaar@chalmers.se
}

electron-density-based formulations, such as the local density approximation [10,11], the semilocal GGA [11,12], or the truly nonlocal vdW-DF [13-16] frameworks. It can be used as a starting point to derive an approximation given in terms of atom-centered pair potentials [17-21]. It can also be evaluated with an explicit orbital basis, for example, as done with calculation in the exact-exchange random phase approximation (RPA) [11,22]. The orbital-based methods are capable of capturing much of the underlying physics of the van der Waals forces [22-24]. Although they come at a much larger computational cost than standard DFT approaches, they are cheap compared to ultra-expensive quantum-chemistry methods, which are mostly used for building benchmarks.

The set of van der Waals density functionals (vdW-DFs) [14-16,25,26] remains entirely within DFT but goes beyond the GGA in approximating the ACF. They include van der Waals forces from first principles, and the exchangecorrelation functional depends only on the density and its gradients in a nonlocal manner. The original general-geometry version is termed simply vdW-DF [15]. A second version termed vdW-DF2 is designed for higher accuracy for atoms and small molecules [25]. These functionals successfully describe sparse matter in its many forms, from clusters of small molecules [27], to the cohesion of layered materials [24,28,29], and adsorption on surfaces [30-33]. vdW-DF has also inspired development of empirical nonlocal correlation functionals [34-37].

The vdW-DF framework develops a description of nonlocal correlations in terms of the semilocal exchange-correlation hole in a single-pole approximation. Standard vdW-DFs build on GGA to account for the (semi)local exchange-correlation energy (in an outer functional) as well for parametrizing the plasmons with an inner functional [25]. While GGA succeeds in describing many kinds of dense matter, its restricted form brings about ambiguities [38]; making for instance some versions better for molecules and others better for solids [39]. Owing to its GGA roots, vdW-DFs inherit some of these ambiguities. This is strikingly so for vdW-DF which generally 
has good binding energies, but whose exchange partner revPBE $[12,14,15,40]$ brings about a chronic overestimation of separations.

Because of this limitation, several alternative exchange partners for vdW-DF correlation have been developed [41-43] and explored [29,44-47]. An example that will be tested here is the nonempirical variant by Cooper called C09 [41].

The inner functional of the nonlocal correlation of $\mathrm{vdW}$ DF relies on the Langreth-Vosko (LV) screened exchange, stemming from a diagrammatic expansion of linear response theory [48]. In vdW-DF2 [25], this functional is replaced with the gradient correction in the large- $Z$ limit to make the functional more appropriate for atoms and molecules [49].

The vdW-DF2 correlation is matched with a slightly refined version of the PW86 exchange functional (PW86r) [50,51], which avoids spurious exchange binding, and accurately reproduces Hartree-Fock exchange for interacting atoms and molecules [51,52]. vdW-DF2 indeed performs well for systems where small molecules are involved [25,31,53,54]. Yet vdWDF has a better asymptotic behavior [55] and tends to produce better adsorption energies for bigger molecules [45,56,57] where many length scales contribute to the binding [57]. These properties indicate that the nonlocal correlation of vdW-DF may have a better transferability across length scales, making it a promising starting point for refining the account of sparse matter. This is especially so if emphasis is on solids [29,43,58] and bigger molecules, because the LV exchange description is appropriate for a slowly varying electron gas.

This paper is motivated by the question: Is the plasmonic description that underpins the vdW-DF account of nonlocal correlation capable of accurately specifying all its functional components? The perspective of having such a unified plasmon representation for the functional form is that one can fully exploit the conservation of the electron response that is built into vdW-DF [15]. To address this question, we here design and test an exchange functional partner to vdW-DF correlation that matches the vdW-DF inner functional to the greatest extent that we deem feasible and relevant.

The total exchange-correlation energy in the vdW-DF framework is given by

$$
\begin{aligned}
E_{\mathrm{xc}}^{\mathrm{vdWDF}} & =\int_{0}^{\infty} \frac{d u}{2 \pi} \operatorname{Tr}[\ln (\nabla \epsilon \cdot \nabla G)]-E_{\mathrm{self}}-\delta E_{\mathrm{xc}} \\
\delta E_{\mathrm{xc}} & =\int_{0}^{\infty} \frac{d u}{2 \pi} \operatorname{Tr} \ln \epsilon-E_{\mathrm{self}}-\underbrace{\left(E_{\mathrm{x}}^{\mathrm{GGA}}+E_{c}^{\mathrm{LDA}}\right)}_{E_{\mathrm{xc}}^{0}} .
\end{aligned}
$$

Here $E_{\text {self }}$ is the self-energy term, $E_{\mathrm{x}}^{\mathrm{GGA}}$ is the GGA exchange energy, and $E_{\mathrm{c}}^{\mathrm{LDA}}$ is the correlation energy in the local density approximation. The term $\delta E_{\mathrm{xc}}$ can compensate for energetic contributions in the semilocal exchange-correlation energy that may be lost with an approximate scalar dielectric function $\epsilon$ [15]. The nonlocal correlation energy in vdW-DF and vdW-DF2 is $E_{c}^{\mathrm{nl}}=E_{\mathrm{xc}}^{\mathrm{vdWDF}}-E_{\mathrm{xc}}^{0}$ expanded to second order in $S=1-1 / \epsilon$. If the inner and outer exchange functionals are the same, $\delta E_{\mathrm{xc}}=0$, the full exchange-correlation energy formally depends purely on a longitudinal projection of the dielectric function and the trivial self-energy
[Eq. (1)]. The exchange-correlation hole is then automatically conserved. ${ }^{1}$

Exact matching between the inner and outer functional for all $s$ is not a good option with the vdW-DF description. The LV form has a poor exchange description in the large- $s$ regime because its enhancement factor is aggressive and, for example, noise sensitive [59]. The value of $E_{\mathrm{c}}^{\mathrm{nl}}$ is specified by the scaled separation $d(\mathbf{r})=q_{0}(\mathbf{r})\left|\mathbf{r}-\mathbf{r}^{\prime}\right|$, where $q_{0}=-4 \pi\left(\varepsilon_{x}^{\mathrm{LV}}+\varepsilon_{\mathrm{c}}^{\mathrm{LDA}}\right)$ depends on the LDA correlation and LV exchange per particle $[15,16,25]$. The rapidly increasing $q_{0} \propto s^{2}$ in the large- $s$ limit implies that scaled separations diverge, tuning out $E_{\mathrm{c}}^{\mathrm{nl}}$. This effect can be interpreted as a soft cutoff. We find that if we in our investigation introduce a hard cutoff at $s=2.5$, we do not greatly affect the nonlocal correlation energy at binding separation. We stress that while introducing a hard cutoff in the non-local correlation part is useful for gauging the role of different $s$ regimes, it is not used in the construction of the new vdW-DF version presented here. Such a hard-cutoff procedure would implicitly break the $f$-sum rule as the contributions from the high- $u$ limit in Eq. (1) are independent of the plasmon model [15,60]. For the outer exchange, we will, based on these observations, let the enhancement factor roll over from the $\mathrm{LV}$ form to that of the PW86r form at $s \approx 2.5$. PW86r [50,51] is chosen for the medium-to-large $s$ regime because it was designed by imposing conservation of the exchange hole arising in the gradient expansion approximation (GEA), and a strong case has been made for its large- $s$ form $[51,52]$. In effect, we bridge the LV form with PW86r creating LV-PW86r.

While we find LV-PW86 a handy and descriptive name for the exchange functional itself, it gets awkward to discuss the full functional in terms of it. We therefore label the corresponding full functional as vdW-DF-cx; cx is short for consistent exchange. The name also emphasize that the new nonempirical vdW-DF-cx makes use of the vdW-DF plasmon-response description [15] for both correlation and exchange. vdW-DF-cx thus secures an exchange-correlation hole conservation for systems where $s<2.5$ is a valid bound on binding contributions from $E_{c}^{\mathrm{nl}}$. This condition is fulfilled for many systems and approximately so for many others.

Figure 1, upper panel, displays the enhancement factor of LV-PW86r exchange functional $F^{\mathrm{LV}-\mathrm{PW} 86 \mathrm{r}}(s)$ alongside corresponding ones for a few other proposed exchange functionals for vdW-DFs. The lower panel displays the derivatives. The total exchange energy is given by

$$
\int d^{3} \mathbf{r} n(\mathbf{r}) \epsilon_{x}^{\mathrm{LDA}}(s) F_{\mathrm{x}}^{\mathrm{LV}-\mathrm{PW} 86 \mathrm{r}}(s) .
$$

The LV-PW86r enhancement factor effectively splines the Langreth-Vosko gradient expansion $[15,48] \quad F^{\mathrm{LV}}(s)=1+$

\footnotetext{
${ }^{1}$ Reference [15], describing the plasmon-pole design of vdWDF, observes that $S=1-1 / \epsilon$ is finite for $\omega \neq 0$ and notes the implications for charge conservation. The $\ln (\nabla \epsilon \nabla G)$ term of Eq. (1) involves a longitudinal projection. Conservation of the corresponding exchange-correlation hole follows because a spatial average (of the hole) constitutes a zero-momentum evaluation. A full presentation is in preparation; details are summarized in http://meetings.aps.org/Meeting/MAR13/Event/183255.
} 


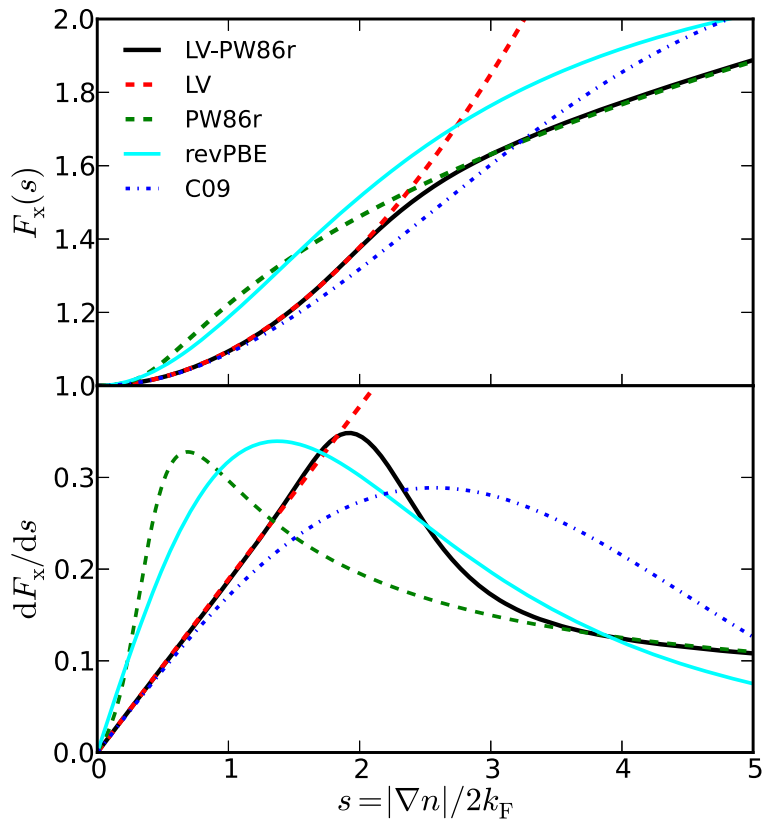

FIG. 1. (Color online) Exchange enhancement factor $F_{x}(s)$ of GGA functionals. The lower panel shows $d F_{x}(s) / d s$ which is important for binding separations. This paper presents the LVPW86r exchange functional (indicated by the thick full curve). This functional is designed as a consistent exchange choice for the vdW-DF correlation. The full functional is labeled vdW-DF-cx.

$\mu_{\mathrm{LV}} s^{2}$, where $\mu_{\mathrm{LV}}=-Z_{\mathrm{ab}} / 9$ and $Z_{\mathrm{ab}}=-0.8491$, with the PW86r exchange enhancement factor $F_{\mathrm{x}}^{\mathrm{PW} 86 \mathrm{r}}(s)=\left(1+a s^{2}+\right.$ $\left.b s^{4}+c s^{6}\right)^{1 / 15}$ as follows:

$$
\begin{aligned}
& F_{x}^{\mathrm{LV}-\mathrm{PW} 86 \mathrm{r}}(s) \\
& \quad=\left(\frac{1}{1+\alpha s^{6}}\right)\left[1+\mu_{\mathrm{LV}} s^{2}\right]+\left(\frac{\alpha s^{6}}{\beta+\alpha s^{6}}\right) F_{\mathrm{x}}^{\mathrm{PW} 86 \mathrm{r}}(s) .
\end{aligned}
$$

The two rational functions inside the brackets tune out the LV form in favor of the PW86r form as $s$ increases. Since these tuning parameters are expressed in terms of sixth powers of $s$, they secure that the inner and outer exchange functional match in the low-to-moderate $s$ regime.

The parameters $\alpha$ and $\beta$ are determined by least-squares fitting of $F_{x}$ to the $1+\mu_{\mathrm{LV}} s^{2}$ form of $\mathrm{LV}$ in the $0<s<2$ region and to that of PW86r in the $4<s<10$ region. An equal quadratic weight to both regions results in $\alpha=0.02178$ and $\beta=1.15$. The choice of these regions entails keeping the $\mathrm{LV}$ form up to about the point where the $F_{\mathrm{x}}^{\mathrm{PW}}{ }^{\mathrm{PWr}}(s)$ form crosses $F_{\mathrm{x}}^{\mathrm{LV}}(s)$.

Figure 2 exemplifies how low-to-moderate $s$ values dominate the nonlocal correlation part of the molecular interaction energy, ${ }^{2} \Delta E_{\mathrm{c}}^{\mathrm{nl}}$. The upper panel shows the fraction of the full $\Delta E_{\mathrm{c}}^{\mathrm{nl}}$ generated as a function of $s_{\text {cutoff }}$ for three members of the

\footnotetext{
${ }^{2}$ This energy is the difference between the nonlocal correlation energy of the full system and that of the reference system(s) $\Delta E_{\mathrm{c}}^{\mathrm{nl}}=E_{\mathrm{c}}^{\mathrm{nl}}[$ main $]-E_{\mathrm{c}}^{\mathrm{nl}}[$ ref $]$. Only density regions $n(\mathbf{r})$ with $s<$ $s_{\text {cutoff }}$ contribute. This $s$ analysis relies on a modification of our in-house vdW-DF code detailed in Refs. [57,61].
}

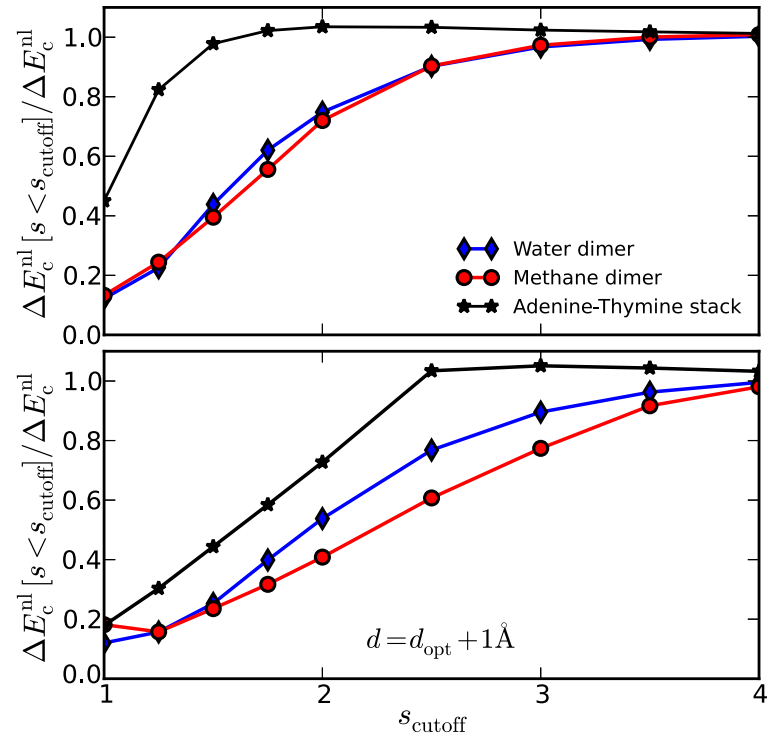

FIG. 2. (Color online) Nonlocal correlation part of interaction energy $\Delta E_{\mathrm{c}}^{\mathrm{nl}}$ at optimal separation and $1 \AA$ beyond for three molecular pairs of the S22 data set as a function of $s_{\text {cutoff }}$ : water dimer, methane dimer, and stacked adenine-thymine pair.

S22 data set [62]: the water-dimer, the methane-dimer, and the stacked adenine-thymine pair at their optimal separation. As $s_{\text {cutoff }}$ increases and we find that most of the energy is accounted for with $s_{\text {cutoff }} \approx 1.5\left(s_{\text {cutoff }} \approx 2.5\right)$, for the stacked aromatic molecules (for the tiny water and methane molecules). ${ }^{3}$ Note that unlike for the nonlocal correlation in vdW-DF, exchange may still contribute significantly to the binding energy for higher $s$ values [51]. The lower panel shows that if the molecules are pulled $1 \AA$ apart (along center-of-mass line), larger $s$ values become more important. Still, density regions with $s<s_{\text {cutoff }} \approx 2.5$ account for the brunt of the energy (though less so for the methane dimer). The upper and lower panels of Fig. 2, taken together, indicate that, with the design, good consistency is achieved at binding separation, but less so for the shape of potential energy curve of tiny molecules somewhat beyond. However, consistency is restored again at larger separations which are dominated by nonlocal correlation because the semilocal correction term $\delta E_{\mathrm{xc}}$ vanishes.

Similar curves as in Fig 2 may also be generated for the total nonlocal correlation energy. We consider that it is the contribution to the binding energy that is most relevant to gauge. Since the binding energy does arise from the difference between the total energy and the reference energy, the curves in Fig. 2 do not necessarily increase monotonically to 1 . This is witnessed in the upper panel in the curve for the Adenine-Thymine stack which reaches a maximum larger than 1 for $s_{\text {cutoff }} \approx 2$. It happens because of somewhat different intramolecular contributions to the nonlocal correlation energy.

\footnotetext{
${ }^{3}$ Reference [57] shows that the nonlocal correlation energy is very sensitive to low-density regions. This is consistent with the presented results when we consider that much of $\Delta E_{\mathrm{c}}$ at binding separation arise from density saddle-point regions between the molecules.
} 


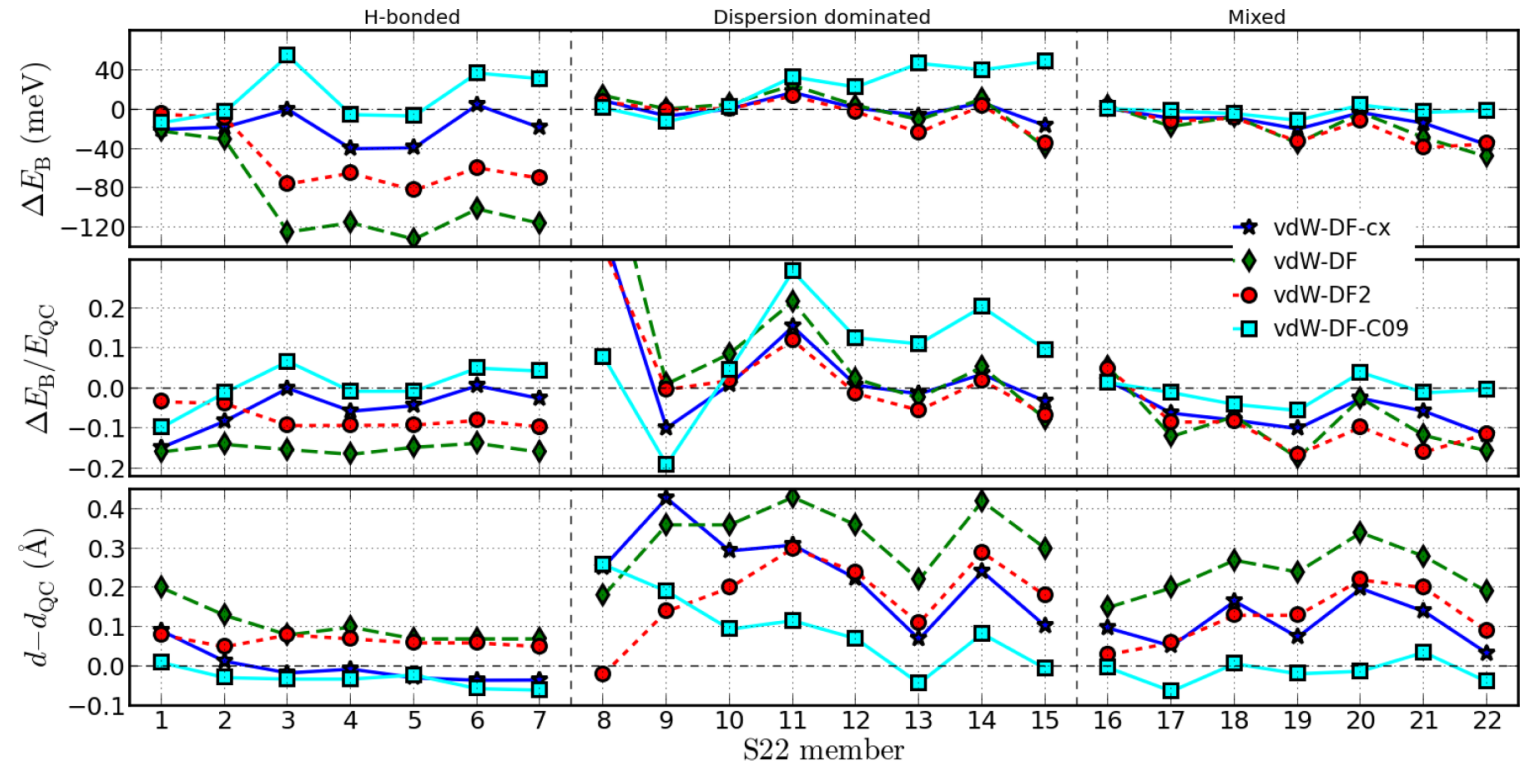

FIG. 3. (Color online) Results for the S22 set of molecular dimers compared with quantum chemistry (QC) results. Enumeration as in Ref. [25]. The top panel compares the energetic deviation $\Delta E_{B}=E^{\mathrm{vdWDF}}-E_{\mathrm{QC}}$, the middle panel the relative energetic deviation, and the bottom panel the deviation from QC separation. The binding energies for the methane dimer (spiking at 8) are 32, 36, 30, and 23 meV for vdW-DF-cx, vdW-DF, vdW-DF2, and QC.

Since the effective cutoff of the nonlocal correlation in vdW-DF is soft, implicit, and system dependent, we can not identify exactly at which $s$ it is natural to tune out the LV form. Even so, because the LV form and PW86r cross at $s \approx 2.5$, hastening or postponing this crossover could lead to a contrived enhancement factor $F_{x}(s)$. A softer crossover is an option, making $F_{x}^{\mathrm{LV}-\mathrm{PW} 86 \mathrm{r}}(s)$ more similar to $F_{x}^{\mathrm{C} 09}(s)$ in the $1<s<3$ region. However, our choice is guided by the wish to use the same plasmonic design in all functional components up to as large $s$ as feasible.

Figure 3 displays our benchmarking ${ }^{4}$ based on the S22 data set of molecular dimers [62] for the proposed functional vdW-DF-cx compared with results of vdW-DF-C09, vdW-DF, and vdW-DF2. Following Ref. [25], the quantum chemistry (QC) result are based on the $\operatorname{CSSD}(\mathrm{T})$ calculations of Ref. [65].

On average, vdW-DF-cx performs marginally better than vdW-DF2. For the binding energy, we find a mean absolute relative deviation of $7 \%$ for both vdW-DF-cx and vdW-DFC09 compared to $9 \%$ for vdW-DF2 5 and $13 \%$ for vdW-DF. For the separations, the mean absolute deviations are $0.13 \AA$ for vdW-DF-cx, $0.06 \AA$ for vdW-DF-C09, $0.14 \AA$ for vdW-DF2, and $0.23 \AA$ for vdW-DF2. Though the overall performances of vdW-DF2 and vdW-DF-cx are similar, their trends differ. $v d W-D F 2$ prevails for small molecules and vdW-DF-cx for the

\footnotetext{
${ }^{4}$ The planewave DFT code QUANTUM ESPRESSO is used [63]. For the S22 calculations, we use a plane-wave cutoff of 50 Ry and density cutoff of 500 Ry. vdW-DF-cx and vdW-DF-C09 calculations rely on ultrasoft pseudopotentials based on PBEsol [38], while vdW-DF and vdW-DF2 rely on PBE. Soler's algorithm is used to evaluate the nonlocal correlation [64].

${ }^{5}$ Reference [25] reports mean absolute relative deviation of $8 \%$ for vdW-DF2.
}

bigger aromatic ones. vdW-DF-C09 clearly has the best overall performance, yet we note that it systematically overestimates the binding energy for the bigger dispersion bound molecules.

The significant overestimation of separations for the methane and ethene dimers ( 8 and 9), when using vdW-DF-cx, we interpret as a consequence of keeping the LV form as long as feasible. LV-PW86r has the largest $d F / d s$ in the $s \approx 2$ region as revealed by the lower panel of Fig. 1. Larger $s$ values matter more for small pointy molecules than for bigger flatter ones, as Fig. 2 exemplifies. This trend can be understood both from the shape as well as from the higher band gap of small molecules [55]. Moreover, the agreements between the outer and the inner functionals are necessarily poorer for these small molecules.

TABLE I. Lattice constants ( $)$ calculated with different versions of vdW-DF.

\begin{tabular}{lccccc}
\hline \hline $\mathrm{xc}$ & vdW-DF & vdW-DF2 & vdW-C09 & vdW-cx & Expt. $^{\mathrm{a}}$ \\
\hline $\mathrm{Li}$ & 3.47 & 3.38 & 3.44 & 3.48 & 3.45 \\
$\mathrm{Na}$ & 4.20 & 4.14 & 4.22 & 4.24 & 4.21 \\
$\mathrm{Al}$ & 4.08 & 4.09 & 4.02 & 4.02 & 4.02 \\
$\mathrm{Cu}$ & 3.70 & 3.74 & 3.58 & 3.57 & 3.57 \\
$\mathrm{Ag}$ & 4.24 & 4.31 & 4.05 & 4.07 & 4.06 \\
$\mathrm{Au}$ & 4.26 & 4.36 & 4.10 & 4.10 & 4.06 \\
$\mathrm{C}$ & 3.59 & 3.62 & 3.56 & 3.56 & 3.54 \\
$\mathrm{Si}$ & 5.50 & 5.54 & 5.43 & 5.43 & 5.42 \\
$\mathrm{~Pb}$ & 5.15 & 5.24 & 4.92 & 4.94 & 4.91 \\
$\mathrm{GaAs}$ & 5.83 & 5.92 & 5.64 & 5.67 & 5.64 \\
$\mathrm{InAs}$ & 6.19 & 6.38 & 6.09 & 6.11 & 6.04 \\
$\mathrm{Pd}$ & 4.01 & 4.09 & 3.88 & 3.89 & 3.88 \\
$\mathrm{MAD}$ & 0.12 & 0.19 & 0.014 & 0.023 & \\
\hline \hline
\end{tabular}

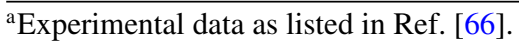


TABLE II. Bulk modulus (GPa) calculated and experimental.

\begin{tabular}{lccccc}
\hline \hline $\mathrm{xc}$ & vdW-DF & vdW-DF2 & vdW-C09 & vdW-cx & Expt. $^{\text {a }}$ \\
\hline $\mathrm{Li}$ & 14.1 & 15.6 & 13.4 & 13.0 & 13.30 \\
$\mathrm{Na}$ & 9.3 & 9.6 & 9.3 & 8.8 & 7.5 \\
$\mathrm{Al}$ & 71 & 65 & 82 & 81 & 79 \\
$\mathrm{Cu}$ & 121 & 105 & 176 & 170 & 142 \\
$\mathrm{Ag}$ & 73 & 64 & 124 & 118 & 109 \\
$\mathrm{Au}$ & 112 & 96 & 182 & 173 & 173 \\
$\mathrm{C}$ & 416 & 394 & 451 & 449 & 443 \\
$\mathrm{Si}$ & 83 & 81 & 76 & 78 & 99 \\
$\mathrm{~Pb}$ & 27 & 25 & 42 & 35 & 39 \\
$\mathrm{GaAs}$ & 75 & 58 & 69 & 59 & 76 \\
$\mathrm{InAs}$ & 62 & 35 & 52 & 38 & 58 \\
$\mathrm{Pd}$ & 140 & 123 & 209 & 207 & 195 \\
$\mathrm{RMAD}$ & 0.19 & 0.30 & 0.12 & 0.12 & \\
\hline \hline
\end{tabular}

$\overline{{ }^{a} \text { Experimental data as listed in Refs. [43,67]. For } \mathrm{Au} \text { [68]. For InAs }}$ [69]. For $\mathrm{Pb}$ [70].

For tightly-bound solids, ${ }^{6}$ Table I confirms our expectations of good lattice constants of vdW-DF-cx. ${ }^{7}$ In an ealier study by Klimeš, Bowler, and Michaelides [43], they investigated the effect of using different vdW-DFs, including variants based on semiempirical fitting [42]. Their benchmarking revealed that vdW-DF and vdW-DF2 typically overestimate lattice constants, especially for heavier elements towards the right end of the periodic system.

As in the work of Cooper [41], Klimeš et al [43], was inspired by the design logic of PBEsol [38] to construct an exchange functional named optB86b as exchange partner for vdW-DF. The logic in C09 and optB86b is to follow the second-order small-s expansion factor $F(s)=1+\mu s^{2}+\cdots$ in a wide region of $s$ values. The lowering of $d F(s) / d s$, relative to PW86r and revPBE, improves lattice constants significantly. LV-PW86r benefits from the same mechanisms as a byproduct of stressing consistency in the plasmon description.

Table II shows the bulk modulus for the solids considered. These are obtained by extracting the second-order coefficient of a fourth-order polynomial fit to total energy for different volumes. The bulk modulus is much improved with vdW-DFcx and C09 compared to vdW-DF and especially vdW-DF2.

Five layered systems, selected for variety and technological interest, provide our final testing ground. Table III presents our results for vdW-DF, vdW-DF2, vdW-DF-C09, and vdW-DFcx, as well as available experimental data and RPA results.

Unfortunately, experimental numbers for interlayer binding energies are scarce. For graphite they vary widely [23] as evidenced by the two examples in the table. Quantum Monte

\footnotetext{
${ }^{6} \mathrm{~A}$ plane-wave cutoff of $50 \mathrm{Ry}$, density cutoff of $500 \mathrm{Ry}$, and k-points sampling of $16 \times 16 \times 16$ is used.

${ }^{7}$ We have also assessed a few atomization energies by calculating the energy for spin polarization at the GGA level, as in Ref. [58]. This procedure indicates that vdW-DF-cx improves the atomization energies over vdW-DF. Sample results in units of eV are (vdW-DF, $v d W-D F-c x, \operatorname{Exp})$ Li: 1.41 1.47, 1.67; Al: 2.95, 3.56, 3.44; Ag: 2.10, 2.96, 2.97
}

Carlo calculations provide a benchmark for graphite [80]. The result of $23 \pm 2 / \AA$ without zero-point motion, appropriate for benchmarking DFT results, falls between the results of vdWDF and vdW-DF-cx.

Björkman and coworkers suggested using results based on RPA [24,79] calculations as a benchmark for sparse-matter methods, and in turn generated a large database of such results for simple layered materials. The lack of experimental data makes it hard to gauge the accuracy of the interlayer binding energies this approach produces, and so does the fact that they kept the in-plane lattice constant fixed. However, RPA predicts excellent lattice separations [24], good elastic coefficients [79], as well as the correct asymptotic scaling laws [23]. While accurate lattice separations could be achieved merely by having a good exchange account [57], accurate elastic coefficients do require a good account of the attractive forces, including the dispersion forces, at least within certain length scales. The good performance of exact-exchange RPA for such layered systems suggests that these results may serve as an additional reference for the characterization of interlayer binding.

For the in-plane lattice constant $a$, trends are similar to those of the regular solids, with vdW-DF-C09 and vdW-DF-cx essentially resolving issues of vdW-DF and vdW-DF2. In the out-of-plane direction, vdW-DF significantly overestimates the lattice constant $c$ as expected. vdW-DF2 improves interlayer separations somewhat, but less than one might expect from the S22 results. Since vdW-DF has a stronger nonlocal correlation effect, we attribute most of this lack of improvement to the fact that the geometry of layered materials is different than for dimers, noting that the distributions of electrons are of central importance [57]. vdW-DF-C09 performs quite well, but underestimates $c$. vdW-DF-cx provides the best results and reduces the vdW-DF-C09 underestimation by roughly a factor of a half.

The interlayer binding energies of our fully relaxed calculations using vdW-DF and vdW-DF2 agree reasonably well with RPA, with the notable expection of $\alpha$-PbO. vdW-DF-cx somewhat overestimates RPA for these systems, though significantly less so than vdW-DF-C09. Björkmann and coworkers [24,79], considering a larger set of layered systems, generally find that vdW-DF and vdW-DF2 underestimate RPA energies. We can speculate that the increase in predicted interlayer binding energy from vdW-DF to vdW-DF-cx does not in general reduce the agreement with RPA.

Comparing predicted elastic coefficients $C_{33}$ to the available experimental data, we find that vdW-DF significantly underestimates the coefficients. This underestimation is reduced with vdW-DF2. vdW-DF-C09 overestimates the coefficients. The agreement between experimental coefficients and those of vdW-DF-cx is excellent, though we note the wide span of predicted experimental results for BN. The vdW-DF-cx results generally agree with the higher end of the experimental results, but differs somewhat from the RPA results.

Finally, we wish to highlight $\alpha-\mathrm{PbO}$. Because the interlayer binding arises from several competing effects, it is challenging to find a good poise between repulsive and attractive contributions. Whereas vdW-DF and vdW-DF2 qualitatively fail, only vdW-DF-cx provides a binding energy that agrees well with that of RPA. 
TABLE III. Lattice constants $(\AA)$, interlayer binding energy per area $\left(\mathrm{meV} / \AA^{2}\right)$, and the $C_{33}$ elastic coefficient calculated with different versions of vdW-DF compared with RPA and experimental data.

\begin{tabular}{|c|c|c|c|c|c|c|}
\hline $\mathrm{xc}$ & vdW-DF & vdW-DF2 & vdW-DF-C09 & vdW-DF-cx & RPA & Expt. \\
\hline \multicolumn{7}{|c|}{ Graphite } \\
\hline$a$ & 2.47 & 2.49 & 2.46 & 2.46 & & $2.46^{\mathrm{a}}$ \\
\hline$c$ & 7.13 & 7.02 & 6.43 & 6.52 & $6.68^{b}$ & $6.68^{\mathrm{a}}$ \\
\hline$E / A$ & 20.7 & 20.1 & 29.3 & 25.2 & $18.8^{\mathrm{c}}$ & $12^{\mathrm{d}} 20^{\mathrm{e}}$ \\
\hline$C_{33}$ & 28 & 35 & 50 & 43 & $36^{\mathrm{b}}$ & $40.7,36.5,38.7,37^{\mathrm{f}}$ \\
\hline \multicolumn{7}{|l|}{$\mathrm{BN}$} \\
\hline$a$ & 2.52 & 2.53 & 2.51 & 2.51 & & $2.51^{\mathrm{g}}$ \\
\hline$c$ & 7.01 & 6.92 & 6.30 & 6.39 & & $6.60^{\mathrm{g}}$ \\
\hline$E / A$ & 20.0 & 19.7 & 28.4 & 24.0 & $14.5^{\mathrm{c}}$ & \\
\hline$C_{33}$ & 25 & 31 & 46 & 38 & $25^{\mathrm{h}}$ & $32.4 \pm 3,35.6,18.7^{\mathrm{f}}$ \\
\hline \multicolumn{7}{|l|}{$\operatorname{MoS} 2$} \\
\hline$a$ & 3.24 & 3.29 & 3.15 & 3.16 & & $3.16^{\mathrm{i}}$ \\
\hline$c$ & 13.13 & 12.89 & 12.15 & 12.27 & & $12.29^{\mathrm{i}}$ \\
\hline$E / A$ & 18.8 & 20.0 & 29.5 & 24.6 & $19.6^{c}$ & \\
\hline$C_{33}$ & 30.2 & 43 & 62 & 51 & $59^{\mathrm{h}}$ & $52^{\mathrm{f}}$ \\
\hline \multicolumn{7}{|l|}{ WSe2 } \\
\hline$a$ & 3.38 & 3.45 & 3.28 & 3.29 & & $3.28^{\mathrm{j}}$ \\
\hline$c$ & 13.95 & 13.77 & 12.91 & 13.01 & & $12.96^{\mathrm{j}}$ \\
\hline$E / A$ & 17.6 & 18.4 & 28.2 & $23.9^{\mathrm{h}}$ & $20.2^{\mathrm{c}}$ & \\
\hline$C_{33}$ & 27 & 38 & 59 & 51 & & $52.1^{\mathrm{k}}$ \\
\hline \multicolumn{7}{|l|}{$\alpha-\mathrm{PbO}$} \\
\hline$a$ & 4.15 & 4.17 & 4.01 & 4.02 & & $3.96^{1}$ \\
\hline$c$ & 5.86 & 5.60 & 4.83 & 4.91 & & $5.01^{1}$ \\
\hline$E / A$ & 12.7 & 13.2 & 25.7 & 20.9 & $20.2^{c}$ & \\
\hline$C_{33}$ & 16 & 24 & 35 & 31 & & \\
\hline
\end{tabular}

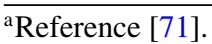

${ }^{\mathrm{b}}$ Reference [23].

${ }^{\mathrm{c}}$ Supplemental Material in Ref. [24].

${ }^{\mathrm{d}}$ Reference [72].

${ }^{\mathrm{e}}$ Reference [73] (low $T$ ).

${ }^{\text {f }}$ See Refs. within Ref. [79].

${ }^{g}$ Reference [74] (low $T$ ).

${ }^{\mathrm{h}}$ Reference [79].

${ }^{\mathrm{i}}$ Reference [75].

${ }^{\mathrm{j}}$ Reference [76].

${ }^{\mathrm{k}}$ Reference [78].

${ }^{1}$ Reference [77].

The results presented here indicate that vdW-DF-cx performs well for solids, layered materials, and aromatic molecules. This is in line with its emphasis on good properties of a slowly varying electron gas. In contrast, vdW-DF2 puts emphasis on small molecules. vdW-DF-cx is constructed to test the ability of the vdW-DF plasmon description to specify all full functional components. The promising results are encouraging for developing van der Waals functionals following a strategy demanding a unified plasmon description.

We thank E. Schröder, P. Erhart, B. I. Lundqvist, and K. Lee for valuable input. The Swedish Research Council (VR) and the Chalmers Area of Advance supported this work. The Swedish National Infrastructure for Computing (SNIC) at the C3SE and HPC2N provided computer time.
[1] X. Wu, M. C. Vargas, S. Nayak, V. Lotrich, and G. Scoles, J. Chem. Phys. 115, 8748 (2001).

[2] S. Grimme, J. Comput. Chem. 25, 1463 (2004).

[3] S. Grimme, J. Comput. Chem. 27, 1787 (2006).

[4] S. Grimme, J. Antony, S. Ehrlich, and H. Krieg, J. Chem. Phys. 132, 154104 (2010).

[5] A. D. Becke and E. R. Johnson, J. Chem. Phys. 123, 154101 (2005).
[6] E. R. Johnson and A. D. Becke, J. Chem. Phys. 124, 174104 (2006).

[7] P. L. Silvestrelli, Phys. Rev. Lett. 100, 053002 (2008).

[8] C. Espejo, T. Rangel, Y. Pouillon, A. Romero, and X. Gonze, Comput. Phys. Commun. 183, 480 (2012).

[9] A. Tkatchenko and M. Scheffler, Phys. Rev. Lett. 102, 073005 (2009). 
[10] O. Gunnarsson and B. I. Lundqvist, Phys. Rev. B 13, 4274 (1976).

[11] D. C. Langreth and J. P. Perdew, Phys. Rev. B 15, 2884 (1977).

[12] J. P. Perdew, K. Burke, and M. Ernzerhof, Phys. Rev. Lett. 77, 3865 (1996).

[13] H. Rydberg, B. I. Lundqvist, D. C. Langreth, and M. Dion, Phys. Rev. B 62, 6997 (2000).

[14] H. Rydberg, M. Dion, N. Jacobson, E. Schröder, P. Hyldgaard, S. I. Simak, D. C. Langreth, and B. I. Lundqvist, Phys. Rev. Lett. 91, 126402 (2003).

[15] M. Dion, H. Rydberg, E. Schröder, D. C. Langreth, and B. I. Lundqvist, Phys. Rev. Lett. 92, 246401 (2004).

[16] T. Thonhauser, V. R. Cooper, S. Li, A. Puzder, P. Hyldgaard, and D. C. Langreth, Phys. Rev. B 76, 125112 (2007).

[17] B. I. Lundqvist, Y. Andersson, H. Shao, S. Chan, and D. C. Langreth, Int. J. Quantum Chem. 56, 247 (1995).

[18] Y. Andersson, D. C. Langreth, and B. I. Lundqvist, Phys. Rev. Lett. 76, 102 (1996).

[19] J. F. Dobson and B. P. Dinte, Phys. Rev. Lett. 76, 1780 (1996).

[20] E. Hult, H. Rydberg, B. I. Lundqvist, and D. C. Langreth, Phys. Rev. B 59, 4708 (1999).

[21] A. Tkatchenko, A. Ambrosetti, and R. A. DiStasio, Jr., J. Chem. Phys. 138, 074106 (2013).

[22] J. Harl and G. Kresse, Phys. Rev. B 77, 045136 (2008).

[23] S. Lebègue, J. Harl, T. Gould, J. G. Ángyán, G. Kresse, and J. F. Dobson, Phys. Rev. Lett. 105, 196401 (2010).

[24] T. Björkman, A. Gulans, A. V. Krasheninnikov, and R. M. Nieminen, Phys. Rev. Lett. 108, 235502 (2012).

[25] K. Lee, E. D. Murray, L. Kong, B. I. Lundqvist, and D. C. Langreth, Phys. Rev. B 82, 081101 (2010).

[26] D. C. Langreth, B. I. Lundqvist, S. D. Chakarova-Käck, V. R. Cooper, M. Dion, P. Hyldgaard, A. Kelkkanen, J. Kleis, L. Kong, S. Li, P. G. Moses, E. Murray, A. Puzder, H. Rydberg, E. Schröder, and T. Thonhauser, J. Phys.: Condens. Matter 21, 084203 (2009).

[27] S. D. Chakarova-Käck, A. Vojvodic, J. Kleis, P. Hyldgaard, and E. Schröder, New J. Phys. 12, 013017 (2010).

[28] J. Rohrer and P. Hyldgaard, Phys. Rev. B 83, 165423 (2011).

[29] E. Londero and E. Schröder, Phys. Rev. B 82, 054116 (2010).

[30] K. Berland, T. L. Einstein, and P. Hyldgaard, Phys. Rev. B 80, 155431 (2009).

[31] K. Lee, Y. Morikawa, and D. C. Langreth, Phys. Rev. B 82, 155461 (2010).

[32] K. Lee, K. Berland, M. Yoon, S. Andersson, E. Schröder, P. Hyldgaard, and B. I. Lundqvist, J. Phys.: Condens. Matter 24, 424213 (2012).

[33] K. Berland, S. D. Chakarova-Käck, V. R. Cooper, D. C. Langreth, and E. Schröder, J. Phys.: Condens. Matter 23, 135001 (2011).

[34] O. A. Vydrov and T. Van Voorhis, J. Chem. Phys. 130, 104105 (2009).

[35] O. A. Vydrov and T. Van Voorhis, Phys. Rev. Lett. 103, 063004 (2009).

[36] O. A. Vydrov and T. Van Voorhis, J. Chem. Phys. 133, 244103 (2010).

[37] T. Björkman, Phys. Rev. B 86, 165109 (2012).

[38] J. P. Perdew, A. Ruzsinszky, G. I. Csonka, O. A. Vydrov, G. E. Scuseria, L. A. Constantin, X. Zhou, and K. Burke, Phys. Rev. Lett. 100, 136406 (2008).
[39] K. Burke, J. Chem. Phys. 136, 150901 (2012).

[40] Y. Zhang and W. Yang, Phys. Rev. Lett. 80, 890 (1998).

[41] V. R. Cooper, Phys. Rev. B 81, 161104 (2010).

[42] J. Klimeš, D. R. Bowler, and A. Michaelides, J. Phys.: Condens. Matter 22, 022201 (2010).

[43] J. Klimeš, D. R. Bowler, and A. Michaelides, Phys. Rev. B 83, 195131 (2011).

[44] A. Gulans, M. J. Puska, and R. M. Nieminen, Phys. Rev. B 79, 201105 (2009).

[45] K. Berland, Ø. Borck, and P. Hyldgaard, Comput. Phys. Commun. 182, 1800 (2011).

[46] I. Hamada and M. Tsukada, Phys. Rev. B 83, 245437 (2011).

[47] G. Graziano, J. Klimeš, F. Fernandez-Alonso, and A. Michaelides, J. Phys.: Condens. Matter 24, 424216 (2012).

[48] D. C. Langreth and S. Vosko, Adv. Quantum. Chem. 21, 175 (1990).

[49] P. Elliott and K. Burke, Can. J. Chem. 87, 1485 (2009).

[50] J. P. Perdew and Y. Wang, Phys. Rev. B 33, 8800 (1986).

[51] É. D. Murray, K. Lee, and D. C. Langreth, J. Chem. Theory Comput. 5, 2754 (2009).

[52] F. O. Kannemann and A. D. Becke, J. Chem. Theory Comput. 5, 719 (2009).

[53] K. Lee, A. K. Kelkkanen, K. Berland, S. Andersson, D. C. Langreth, E. Schröder, B. I. Lundqvist, and P. Hyldgaard, Phys. Rev. B 84, 193408 (2011).

[54] D. Chen, W. A. Al-Saidi, and J. K. Johnson, J. Phys.: Condens. Matter 24, 424211 (2012).

[55] O. A. Vydrov and T. Van Voorhis, Phys. Rev. A 81, 062708 (2010).

[56] J. Björk, F. Hanke, C.-A. Palma, P. Samori, M. Cecchini, and M. Persson, J Phys. Chem. Lett. 1, 3407 (2010).

[57] K. Berland and P. Hyldgaard, Phys. Rev. B 87, 205421 (2013).

[58] E. Ziambaras, J. Kleis, E. Schröder, and P. Hyldgaard, Phys. Rev. B 76, 155425 (2007).

[59] E. Londero and E. Schröder, Comput. Phys. Commun. 182, 1805 (2011).

[60] D. C. Langreth and B. I. Lundqvist, Phys. Rev. Lett. 104, 099303 (2010).

[61] K. Berland and P. Hyldgaard, J. Chem. Phys. 132, 134705 (2010).

[62] P. Jurečka, J. Šponer, J. Černý, and P. Hobza, Phys. Chem. Chem. Phys. 8, 1985 (2006).

[63] P. Giannozzi, S. Baroni, N. Bonini, M. Calandra, R. Car, C. Cavazzoni, D. Ceresoli, G. L. Chiarotti, M. Cococcioni, I. Dabo, A. D. Corso, S. de Gironcoli, S. Fabris, G. Fratesi, R. Gebauer, U. Gerstmann, C. Gougoussis, A. Kokalj, M. Lazzeri, L. Martin-Samos, N. Marzari, F. Mauri, R. Mazzarello, S. Paolini, A. Pasquarello, L. Paulatto, C. Sbraccia, S. Scandolo, G. Sclauzero, A. P. Seitsonen, A. Smogunov, P. Umari, and R. M. Wentzcovitch, J. Phys.: Condens. Matter 21, 395502 (2009).

[64] G. Román-Pérez and J. M. Soler, Phys. Rev. Lett. 103, 096102 (2009).

[65] L. Grafova, M. Pitoňak, J. Řezač, and P. Hobza, J. Chem. Theory Comput. 6, 2365 (2010).

[66] P. Haas, F. Tran, and P. Blaha, Phys. Rev. B 79, 085104 (2009).

[67] G. I. Csonka, J. P. Perdew, A. Ruzsinszky, P. H. T. Philipsen, S. Lebègue, J. Paier, O. A. Vydrov, and J. G. Ángyán, Phys. Rev. B 79, 155107 (2009). 
[68] G. Cordoba and C. R. Brooks, Phys. Status Solidi A 6, 581 (1971).

[69] S. W. Ellaway and D. A. Faux, J. Appl. Phys. 92, 3027 (2002).

[70] Y. K. Vohra and A. L. Ruoff, Phys. Rev. B 42, 8651 (1990).

[71] Y. Baskin and L. Meyer, Phys. Rev. 100, 544 (1955).

[72] Z. Liu, J. Z. Liu, Y. Cheng, Z. Li, L. Wang, and Q. Zheng, Phys. Rev. B 85, 205418 (2012).

[73] R. Zacharia, H. Ulbricht, and T. Hertel, Phys. Rev. B 69, 155406 (2004).

[74] W. Paszkowicz, J. Pelka, M. Knapp, T. Szyszko, and S. Podsiadlo, Appl. Phys. A 75, 431 (2002).
[75] K. D. Bronsema, J. L. De Boer, and F. Jellinek, Z. Anorg. Allgem. Chem. 540, 15 (1986).

[76] W. Schutte, J. D. Boer, and F. Jellinek, J. Solid State Chem. 70, 207 (1987).

[77] J. Leciejewicz, Acta Crystallogr. 14, 1304 (1961).

[78] Y. Zhao, X. Luo, H. Li, J. Zhang, P. T. Araujo, C. K. Gan, J. Wu, H. Zhang, S. Y. Quek, M. S. Dresselhaus, and Q. Xiong, Nano Lett. 13, 1007 (2013).

[79] T. Björkman, A. Gulans, A. V. Krasheninnikov, and R. M. Nieminen, J. Phys.: Condens. Matter 24, 424218 (2012).

[80] L. Spanu, S. Sorella, and G. Galli, Phys. Rev. Lett. 103, 196401 (2009). 